\title{
Uncountable locally free groups and their group rings
}

\author{
TSUNEKAZU NISHINAKA \\ University of Hyogo \\ 8-2-1 Gakuen Nishimachi Nishiku \\ Kobe-City 651-2197 Japan \\ Email: nishinaka@econ.u-hyogo.ac.jp
}

\begin{abstract}
In this note, we show that an uncountable locally free group, and therefore every locally free group, has a free subgroup whose cardinality is the same as that of $G$. This result directly improve the main result in [4] and establish the primitivity of group rings of locally free groups.
\end{abstract}

\section{INTRODUCTION}

A group $G$ is called locally free if all of its finitely generated subgroups are free. As a consequence of Nielsen-Schreier theorem, a free group is always locally free. If the cardinality $|G|$ of $G$ is countable, then $G$ is locally free if and only if $G$ is an ascending union of free groups. In particular, $G$ is a locally free group which is not free provided that it is a properly ascending union of non-abelian free groups of bounded finite rank. In fact, in this case, $G$ is infinitely generated and Hopfian and so it is not free (also see [5] and [1]). If $|G|$ is uncountable, that is $|G|>\aleph_{0}$, then it was studied in the context of almost free groups, and it is also known that there exists an uncountable locally free group which is not free ([2]).

Now, clearly, if $G$ is a locally free group with $|G|=\aleph_{0}$, then $G$ has a free subgroup whose cardinality is the same as that of $G$. In the present note, we shall show that it is true for locally free groups of any cardinality. In fact, we shall prove the following theorem:

Theorem 1.1. If $G$ is a locally free group with $|G|>\aleph_{0}$, then for each finitely generated subgroup $A$ of $G$, there exists a subgroup $H$ of $G$ with $|H|=|G|$ such that $A H \simeq A * H$, the free product of $A$ and $H$.

In particular, $G$ has a free subgroup of the same cardinality as that of $G$.

2000 Mathematics Subject Classification: 20E05, 20E25, 20C07

Key words and phrases: uncountable locally free group, primitive group ring.

This research was partially supported by Grants-in-Aid for Scientific Research (KAKEN) under grant no. 26400055 
Theorem 1.1 means that there is no need to assume the existence of free subgroups in [4, Theorem 1]. That is, we can improve the theorem and establish the primitivity of group rings of locally free groups, where a ring $R$ is (right) primitive provided it has a faithful irreducible (right) $R$-module.

\section{Proof of the THEOREM}

In order to prove Theorem 1.1, we prepare necessary notations and some lemmas which include a result due to Mal'cev [3]. Some of them might be trivial for experts but we include their proofs for completeness.

For a finitely generated subgroup $H$ of a locally free group $G, \mu_{G}(H)$ is defined to be the least positive integer $m$ such that $H \subseteq F_{m}$ for some free subgroup $F_{m}$ of rank $m>0$ in $G$. The rank $r(G)$ of $G$ is defined to be the maximum element in

\section{$\left\{\mu_{G}(H) \mid H\right.$ is a finitely generated subgroup in $\left.G\right\}$}

or $r(G)=\infty$. We should note that $r(G)$ is finite if and only if for each finitely generated subgroup $H$ of $G$, there exists a free subgroup $N$ of rank $r(G)$ such that $H \subseteq N$. We should also note that for subgroups $H, M$ and $N$ of $G$ with $H \subseteq M \subseteq N, \mu_{M}(H) \geq \mu_{N}(H)$ holds.

Lemma 2.1. Let $G$ be a locally free group, $D$ a finitely generated subgroup of $G$ with $\mu_{G}(D)=m$ and $M$ a subgroup of $G$ such that $D \subseteq M$ and $r(M)=m$. For $g \in G \backslash M$, set $M_{g}=M\langle g\rangle$; the subgroup of $G$ generated by $g$ and the elements in $M$, and let $r\left(M_{g}\right)=n$.

Then, if $n>m$, then $n=m+1$ and there exists a free subgroup $F_{m}$ of rank $m$ in $M$ such that $D \subseteq F_{m}$ and $F_{m}\langle g\rangle$ is isomorphic to the free product $F_{m} *\langle g\rangle$.

Proof. Since $r\left(M_{g}\right)=n$, there exists a finitely generated subgroup $C$ of $M_{g}$ such that $\mu_{M_{g}}(C)=n$. Since $C$ is finitely generated, it can be easily seen that there exists finite number of elements $a_{1}, \ldots, a_{l}$ in $M$ such that $C \subseteq\left\langle a_{1}, \ldots, a_{l}, g\right\rangle$. We have then that there exists a free subgroup $F_{m}$ of rank $m$ in $M$ such that $\left\langle a_{1}, \ldots, a_{l}\right\rangle D \subseteq F_{m}$ because of $r(M)=m$. Since $C \subseteq F_{m}\langle g\rangle$ and $\mu_{M_{g}}(C)=n$, we see that $r\left(F_{m}\langle g\rangle\right) \geq n$. On the other hand, $r\left(F_{m}\langle g\rangle\right) \leq m+1$ because of $g \notin F_{m}$. Combining these with the assumption $n>m$, we get that $n=m+1=r\left(F_{m}\langle g\rangle\right)$, which implies $F_{m}\langle g\rangle \simeq F_{m} *\langle g\rangle$.

For a locally free group of finite rank, the following result due to Mal'cev is well-known.

Lemma 2.2. (See [3]) If $G$ is a locally free group of finite rank, then the cardinality of $G$ is countable; namely $|G|=\aleph_{0}$. 
On the other hand, if $G$ is not of finite rank, then we have the following property:

Lemma 2.3. If $G$ is a locally free group whose rank is not finite, then for each finitely generated subgroup $A$ of $G$, there exists an element $x \in G$ with $x \notin A$, such that $A\langle x\rangle \simeq A *\langle x\rangle$, the free product of $A$ and $\langle x\rangle$.

Proof. Let $A$ be a finitely generated subgroup of $G$. We have then that $A$ is a free group of finite rank, because $G$ is locally free. Since the rank of $G$ is not finite, there exists a free subgroup $F$ of $G$ such that $A \subsetneq F$ and $r(F)>r(A)$. Let $A=\left\langle y_{1}, \ldots, y_{l}\right\rangle$ and $F=\left\langle x_{1}, \ldots, x_{m}\right\rangle$, where $l=r(A)$ and $m=r(F)$. If for $A_{1}=\left\langle y_{1}, \ldots, y_{l}, x_{1}\right\rangle, r\left(A_{1}\right)=l+1$, then $A_{1} \simeq A *\left\langle x_{1}\right\rangle$. If $r\left(A_{1}\right) \leq l$ then there exists $i \in\{2, \ldots, m\}$ such that $r\left(A_{i}\right)=r\left(A_{i-1}\right)+1$, where $A_{i}=\left\langle y_{1}, \ldots, y_{l}, x_{1}, \ldots, x_{i}\right\rangle$. We have then that $A_{i} \simeq A_{i-1} *\left\langle x_{i}\right\rangle$. Since $A \subseteq A_{i-1}$, we have thus seen that $A\langle x\rangle \simeq A *\langle x\rangle$ for some $x \in G \backslash A$.

Let $G=A * B$ be the free product of $A \neq 1$ and $B \neq 1$. Clearly, if $|G|=\aleph_{0}$, then $G$ has a free subgroup whose cardinality is the same as that of $G$. If $|G|>\aleph_{0}$, then either $|A|=|G|$ or $|B|=|G|$, say $|A|=|G|$. Let $I$ be a set with $|I|=|A|$, and for each $i \in I$, let $a_{i}$ be in $A$ such that $a_{i} \neq a_{j}$ for $i \neq j$. We have then that for $1 \neq b \in B$, the elements $\left(a_{i} b\right)^{2}$ over $i \in I$ freely generate the subgroup of $G$ whose cardinality is the same as that of $G$. Hence we have

Lemma 2.4. If $G=A * B$ is the free product of $A$ and $B$, then $G$ has a free subgroup whose cardinality is the same as that of $G$.

We are now read to prove Theorem 1.1.

Proof of Theorem 1.1. Let $A$ be a finitely generated subgroup of $G$. We set

$$
\mathcal{B}=\{B \mid B \text { is a non-trivial subgroup of } G \text { such that } A B \simeq A * B\} .
$$

Since $G$ is locally free, $A$ is a free group of finite rank. By assumption, $|G|>\aleph_{0}$, and so the rank of $G$ is not finite by Lemma 2.2. Hence, by Lemma 2.3, there exists an element $g \in G \backslash A$ such that $A\langle g\rangle \simeq A *\langle g\rangle$, whence $\langle g\rangle \in \mathcal{B}$; thus $\mathcal{B} \neq \emptyset$. Let $B_{1} \subseteq B_{2} \subseteq \cdots \subseteq B_{i} \subseteq \cdots$ be a chain of $B_{i}$ 's in $\mathcal{B}$, and let $B^{*}=\bigcup_{i=1}^{\infty} B_{i}$. We can see that $B^{*}$ belongs to $\mathcal{B}$. In fact, if not so, then $A B^{*} \not \mathcal{\sim} A * B^{*}$, and so there exists a finitely generated subgroup $C$ of $B^{*}$ such that $A C \not A * C$. However, because $C$ is finitely generated in $B^{*}$, we have $C \subseteq B_{i}$ for some $i$, which implies $A B_{i} \not 千 A * B_{i}$, a contradiction. We have thus shown that $(\mathcal{B}, \subseteq)$ is an inductively ordered set. By Zorn's lemma, there exists a maximal 
element $H$ in $(\mathcal{B}, \subseteq)$. We shall show $|H|=|G|$, which completes the proof of the theorem. In fact, $A H \simeq A * H$ and it has also a free subgroup whose cardinality is the same as that of $G$ by Lemma 2.4.

Suppose, to the contrary, that $|H|<|G|$. Set $N=A H(\simeq A * H)$, and for a finitely generated subgroup $D$ of $N$ with $\mu_{G}(D)=m$, let $\mathcal{M}(D)$ be the set of subgroups $M$ of $G$ such that $D \subseteq M$ and $r(M)=m$. We can see that $(\mathcal{M}(D), \subseteq)$ is an inductively ordered set as follows: Since $m=\mu_{G}(D)$, there exists a free subgroup $F_{m}$ of rank $m$ in $G$ such that $D \subseteq F_{m}$. Hence $F_{m} \in \mathcal{M}(D)$, whence $\mathcal{M}(D) \neq \emptyset$. Let $M_{1} \subseteq$ $M_{2} \subseteq \cdots \subseteq M_{i} \subseteq \cdots$ be a chain of $M_{i}$ 's in $\mathcal{M}$, and let $M^{*}=\bigcup_{i=1}^{\infty} M_{i}$. Clearly, $D \subseteq M^{*}$. By the definition of the rank, $r\left(M^{*}\right) \geq \mu_{M^{*}}(D)$. Since $M^{*} \subseteq G$, we have that $\mu_{M^{*}}(D) \geq \mu_{G}(D)=m$; thus $r\left(M^{*}\right) \geq m$. On the other hand, by the definition of $r\left(M^{*}\right)$, there exists a finitely generated subgroup $C$ of $M^{*}$ such that $\mu_{M^{*}}(C)=r\left(M^{*}\right)$. Since $C$ is finitely generated, there exists $i>0$ such that $C \subseteq M_{i}$, and then $\mu_{M_{i}}(C) \leq r\left(M_{i}\right)$, which implies that $r\left(M^{*}\right)=\mu_{M^{*}}(C) \leq \mu_{M_{i}}(C) \leq$ $r\left(M_{i}\right)=m$; thus $r\left(M^{*}\right) \leq m$. Hence we have $r\left(M^{*}\right)=m$. We have thus proved that $M^{*} \in \mathcal{M}(D)$ and that $(\mathcal{M}(D), \subseteq)$ is an inductively ordered set.

Again by Zorn's lemma, there exists a maximal element $M(D)$ in $(\mathcal{M}(D), \subseteq)$. Let $L=\bigcup_{D \in \mathcal{D}} M(D)$, where $\mathcal{D}$ is the set consisting of all finitely generated subgroups of $N$. Since $r(M(D))$ is finite for each $D \in \mathcal{D}$, it follows from Lemma 2.2 that $|M(D)|=\aleph_{0}$ for each $D \in \mathcal{D}$. Hence we have $|L|<|G|$ because $|\mathcal{D}|=|N|<|G|$. In particular, there exists $g \in G$ such that $g \notin L$. Note that $g \notin N$ because of $N \subset L$, and so $g \notin H$. We shall show that $N\langle g\rangle \simeq N *\langle g\rangle$. In order to do this, it suffices to show that for each $D \in \mathcal{D}, D\langle g\rangle \simeq D *\langle g\rangle$ holds.

Let $r(M(D))=m$ for $D \in \mathcal{D}$ and let $M_{g}=M(D)\langle g\rangle$. Since $D \subseteq M_{g}$ and $\mu_{M_{g}}(D) \geq \mu_{G}(D)=m$, it follows that $r\left(M_{g}\right) \geq m$. Moreover, since $g \notin M(D)$, we have $M(D) \subsetneq M_{g}$. Hence the maximality of $M(D)$ implies $r\left(M_{g}\right)>m$. It follows from Lemma 2.1 that there exists a free subgroup $F_{m}$ of rank $m$ in $M(D)$ such that $D \subseteq F_{m}$ and $F_{m}\langle g\rangle \simeq F_{m} *\langle g\rangle$. Hence we have $D\langle g\rangle \simeq D *\langle g\rangle$.

We have thus shown that $N\langle g\rangle \simeq N *\langle g\rangle$, which contradicts the maximality of $H$ because $N *\langle g\rangle=A *(H *\langle g\rangle)$. This completes the proof of the theorem.

Theorem 1.1 shows that the assumption on existence of free subgroups in [4, Theorem 1] can be dropped. That is, we have the following theorem: 
Theorem 2.5. Let $G$ be a non-abelian locally free group. If $R$ is a domain with $|R| \leq|G|$, then $R G$ is primitive.

In particular, $K G$ is primitive for any field $K$.

\section{REFERENCES}

[1] G. Higman, A finitely related group with an isomorphic factor group, J. London Math.Soc., 26(1951), 59-61

[2] G. Higman, Almost free groups, Proc. London Math.Soc., 1(3)(1951), 284-290

[3] A. I. Mal'cev, On groups of finite rank, Mat. Sbornik N.S., 22(64)(1948), 351352.

[4] T. Nishinaka, Group rings of countable non-abelian locally free groups are primitive, Int. J. algebra and computation, 21(3) (2011), 409-431

[5] M. Takahashi, Note on locally free groups, Osaka, City, Univ., 1(1950), 65-70. 